-Clinical Research.

\title{
Application of anterolateral thigh free flap to reconstruct head and neck soft tissue defects
}

\author{
Wei-Wei Liu, 1,2 Xue-Kui Liu, ,1,2 Zhu-Ming Guo, ,1,2 Hao Li, 1,2 Qiu-Li Li , 1,2 \\ Quan Zhang ${ }^{1,2}$ and An-Kui Yang ${ }^{1,2}$
}

\author{
1. State Key Laboratory of \\ Oncology in South China, \\ Guangzhou, Guangdong, 510060, \\ P. R. China \\ 2. Department of Head \\ and Neck Surgery, \\ Sun Yat-sen University \\ Cancer Center, \\ Guangzhou, Guangdong, 510060 , \\ P. R. China
}

Correspondance to: Wei-Wei Liu

$$
\begin{aligned}
& \text { Tel.: 86.20.87343302 } \\
& \text { E-mail:drliuww@gmail.com }
\end{aligned}
$$

\footnotetext{
This paper was translated into English from its original publication in Chinese. Translated by:Lian-Yuan Tao and Hua He

The original Chinese version of this paper is published in:Ai Zheng (Chinese Journal of Cancer) 28(10); http://www.cjcsysu.cn/ cn/article.asp?id=15677 )
}

Submitted : 2008-11-20

Revised : 2009-05-03
[Abstract] Background and Objective: Although anterolateral thigh (ALT) flap has the advantage of limited damage to the donor site, its clinical application is not common. This study was to study the clinical value of ALT flap in the reconstruction of head and neck soft tissue defects. Methods: Twenty patients receiving reconstruction of head and neck soft tissue defects using ALT flap at Sun Yat-sen University Cancer Center between November 2004 and May 2008 were retrospectively reviewed. Surgical procedures in the harvest of ALT flap were described. The surgical anatomy and successful rate were reported. Results: Twenty patients were all alive. The vessel pedicle length of ALT flap ranged from $5 \mathrm{~cm}$ to $14 \mathrm{~cm}$, with an average of $9.9 \mathrm{~cm}$. The harvested ALT flap was (4-9) $\mathrm{cm} \times(6-16) \mathrm{cm}$. The skin perforator was classified into four cases of intermuscular septal branch $(20 \%)$ and 16 cases of musculocutaneous branch $(80 \%)$. The head and neck soft tissue defects included 14 cases (nine cases of tongue and oral floor, three cases of buccal mucosas, one case of hard palate, and one case of retromolar trigone mucosa) intraoral mucosa and six cases of facial-cervical skin defects. Except for one case, the donor sites were primarily sutured. Conclusion: ALT flap is one of valuable and potential donor site for the reconstruction of head and neck soft tissue defects. It could achieve a high successful rate and causes little influence to the donor sites.

Key words: surgical flaps, reconstructive surgical procedures, anterolateral thigh flap, head and neck neoplasms

Head and neck soft tissue defects are commonly reconstructed using forearm free flap, but this needs to sacrifice the major forearm blood vessels and perform skin grafting for the donor site, which would damage the shape and the function of the donor site. Anterolateral thigh (ALT) flap has been gradually applied clinically since the 90 s as a new free flap donor sit. The skin blood supply of ALT flap is usually from the skin perforators of the descending branch of lateral femoral circumflex artery, which belongs to the perforator flap., ${ }^{1,2}$ Compared with the forearm flap, ALT flap do not need to cut off the main vessels, so that the donor site can be sutured directly. Therefore, ALT flap has become a major donor site for repairing the head and neck soft tissue defects in Taiwan nowadays. $^{3}$ The application of ALT flap has also been increasingly reported in the United States and Europe. ${ }^{4-8}$ However, because of the anatomic variation of the perforators in ALT flap, such technology has not yet been applied widely in China, and has been seldom reported. We 
believe that, as a main potential donor site for the reconstruction of head and neck soft tissue defects, ALT flap has its unique characteristics that it seldom affects the donor area. We retrospectively reviewed the clinic data of 20 cases with soft tissue defects in head and neck whose defects were reconstructed using ALT flap in our hospital, focusing on surgical technology and short-term results.

\section{Data and Methods}

Clinical data. he clinic data of patients undergoing reconstructive operation with free tissue flap at the Head and Neck Surgery, Sun Yat-sen University Cancer Center from November 2004 to May 2008 were retrospectively analyzed. Twenty patients (15 males, five females) received reconstructive operation with ALT flap, aged from 19 to 71 years with a mean age of 49 years. Among them, 13 cases had squamous cell carcinoma in oral cavity or oral pharynx (eight cases in tongue, three cases in bucca, one case in retromolar trigone, and one case in tongue base), one case had face skin squamous cell carcinoma, three cases had soft tissue sarcoma in face, two cases had sarcoma of maxilla, and one case had recurrent laryngeal carcinoma. All patients underwent primary radical resection and reconstruction using free ALT flap. All patients were followed-up for at least six months.

Surgical operation. The perforators of ALT flap are mapped by a high-frequency color Doppler ultrasound

Flap design: Resection of head and neck primary tumor and harvesting of the ALT flap were performed simultaneously. The patient was in a supine position. Firstly, the marking line was drawn between the anterior superior iliac spine (ASIS) and the upper anterolateral border of the patella (iliac patellar line). Secondly, the mid-point of inguinal ligament (femoral artery pulsating site) to the midpoint of iliac patellar was linked by a line, which marked the projection of the descending branch of lateral circumflex femoral artery on the body surface. A circle, centered in the midpoint of iliac line, was drawn with a radius of $3 \mathrm{~cm}$, which covered the possible corresponding skin site of the perforator vessels. Flap designing was based on the estimated size and shape of the defect along the direction of iliac patellar line. Generally, the harvested flap was designed to have a spindle -like shape. The

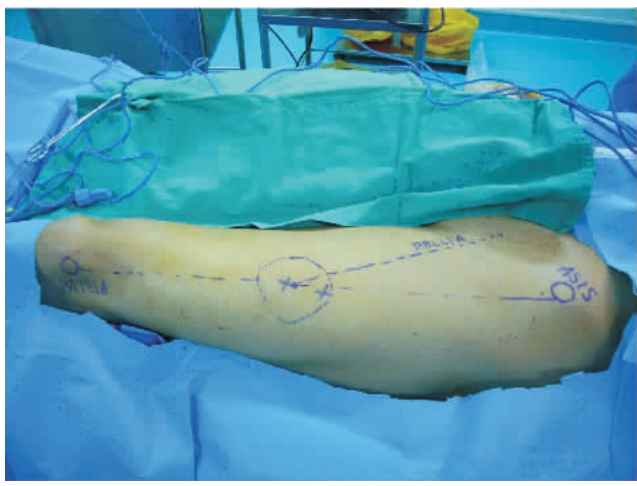

Figure 1 Localization of anterolateral thigh perforators according to the body surface symbol

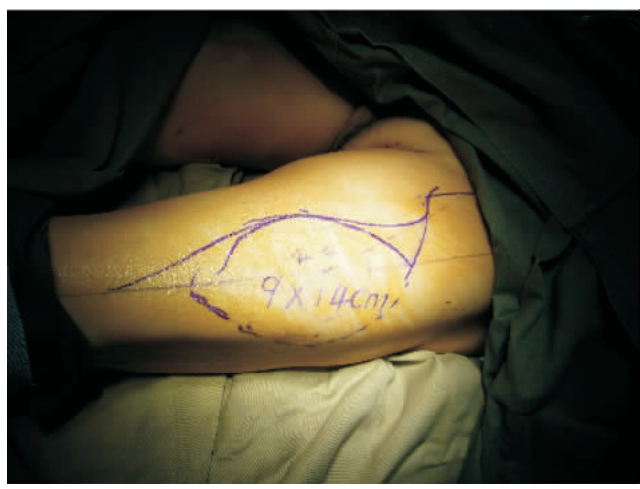

Figure 2 Design of the anterolateral thigh flap according to the iliopatelar line and the marking of the harvested area

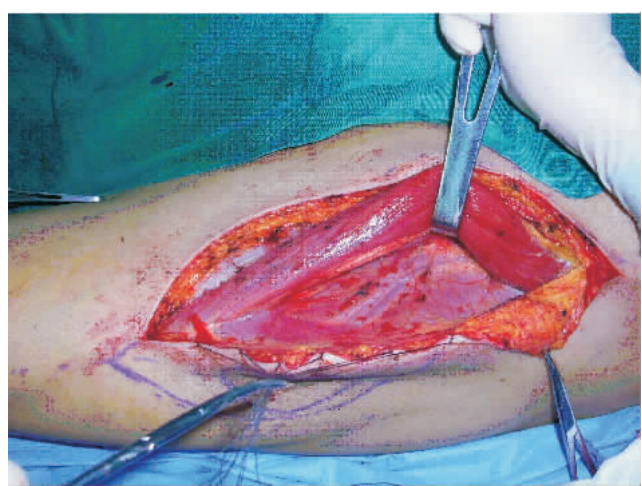

Figure 3 Exploration of the descending branches of the lateral circumflex femoral artery and its perforators

perforators had to be located in the middle $1 / 3$ part of the flap. The specific size of the flap was decided according to the virtual defects after primary tumor resection(Fig. 1-6).

Locating and exploration of perforator vessels: The application of tourniquet should be avoided during surgical procedures. Firstly, the skin incision was made along the inside edge of the flap, and the subfascial dissection was continued down to the 


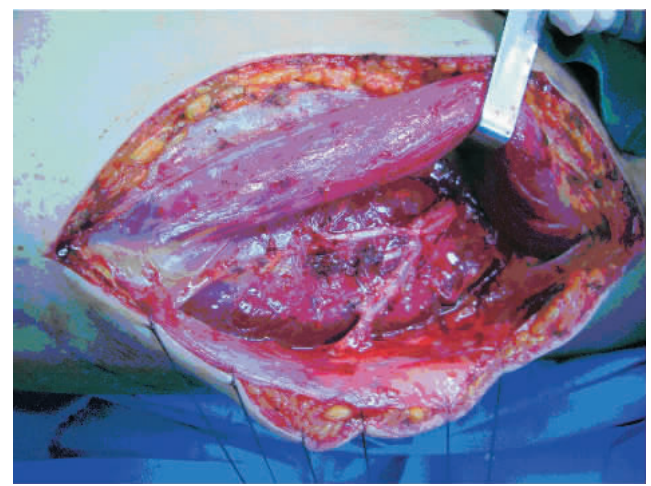

Figure 4 Freeing of the anterolateral thigh perforators and protecting accompanying lateral femoral nerve

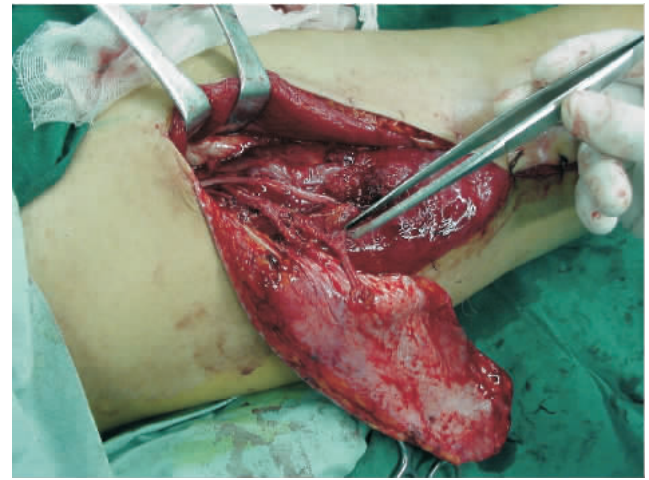

Figure 5 Freeing the vessel pedicle retrogressively and harvesting the ALT flap

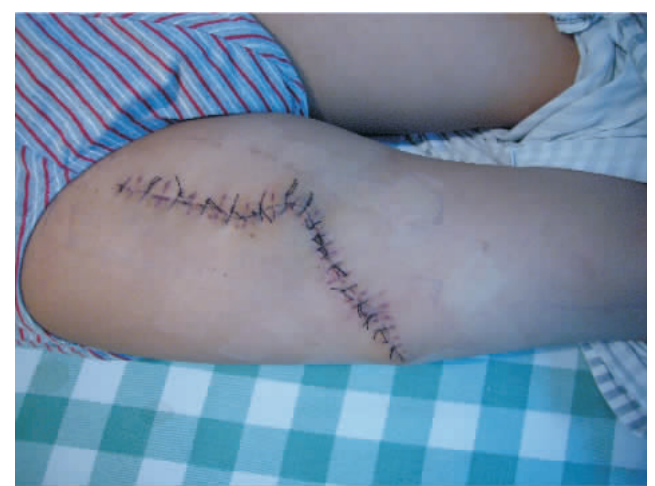

Figure 6 Primarily suturing the donor wound of ALT flap

fascia lata with monopolar diathermy. Bleeding points was stopped by bipolar coagulation. Fascia lata was incised to expose the femoral rectus. The flap was developed under facia lata until the junction of the vastus lateralis and the rectus femoralis. The intermuscular space was opened and the descending branch of lateral circumflex femoral artery was explored. The type of the perforator vessels was firstly determined. For septum intermuscular perforators, the perforator vessels could be seen originating from the muscular septum on the surface of vastus lateralis. However, the majority of them were musculocutaneous perforators, which went through the lateral femoralis muscle. Then this muscle should be partially resected until the exposure of the perforators. Meanwhile, the position of the musculocutaneous perforators should be located.

Dissection of free vascular pedicle: After the anatomy of perforators was clear, dissection was made forward and backward. A small amount of muscle sleeve should be preserved to prevent vascular injury when freeing perforator vessels near the skin. The small vessel in the muscle should be ligated. After exposure of the lateral femoral nerve, the nerve from the vascular pedicle was separated for protection. Vascular pedicle vessels were lifted by vascular gallus, and were freed retrogradely toward the proximal part of the descending branch of lateral circumflex femoral artery until the beginning part of the deep femoral artery. Vascular pedicle, which contained an artery and two veins from the proximal part, was marked. After freeing the vascular pedicle, $2 \%$ lidocaine gauze was applied to prevent possible spasm during the freeing course.

Excision and installation of flap, and microvascular anastomosis: the size and shape of defects were determined after the resection of the primary tumor. The flap was harvested corresponding to the size of the defects. The flap was installed to the defect site and fixed by suture, followed by microvascular anastomosis. The choice of vessels in the recipient site should be based on a number of factors, including the matched size of donor vessels, vascular quality of the recipient site, defect location, as well as the length of the artery pedicle. Arteria thyreoidea superior, arteria facialis or transverse cervical artery are the common choices. First, endto-end anastomosis of the arteries was carried out. External jugular vein or internal jugular vein was chosen to perform end -to -end or end -to -side anastomosis. Patients undergoing neck dissection should preserve the inferior segment of external jugular vein or internal jugular vein for microvascular anastomosis. For patients who had oral soft tissue defects, their vascular pedicles were drawn through the tunnel of mouth floor to the neck for anastomosis. Sufficient width of the tunnel was needed to make sure that vascular pedicel was out of pressure. 
Table 1 Reconstruction of head and neck soft tissue defects using free ALT flap $(n=20)$

\begin{tabular}{|c|c|c|c|c|c|c|c|}
\hline Case & Sex & Age & Primary disease & Defects & Flap size $(\mathrm{cm})$ & Pedicle length $(\mathrm{cm})$ & Peforator type \\
\hline 1 & Male & 68 & Facial skin SCC & Facial skin & $8 \times 13$ & 12 & IMSP \\
\hline 2 & Male & 51 & Maxillary sarcoma & Facial skin & $5 \times 8$ & 14 & MCP \\
\hline 3 & Female & 34 & Buccal SCC & Buccal mucosa & $5.5 \times 8$ & 11 & IMSP \\
\hline 4 & Female & 40 & Tongue SCC & Tongue and FOM & $5 \times 6$ & 11 & MCP \\
\hline 5 & Mele & 55 & Buccal SCC & Buccal mucosa & $4 \times 6$ & 12 & IMSP \\
\hline 6 & Female & 42 & Tongue SCC & Tongue and FOM & $4.5 \times 8$ & 10 & MCP \\
\hline 7 & Male & 20 & Maxillary sarcoma & Hard palate & $5 \times 7$ & 8 & MCP \\
\hline 8 & Male & 31 & Facial soft tissue sarcoma & Facial skin & $9 \times 16$ & 13 & MCP \\
\hline 9 & Male & 49 & Facial soft tissue sarcoma & Facial skin & $7 \times 7$ & 5 & MCP \\
\hline 10 & Male & 59 & Buccal SCC & Buccal mucosa & $5 \times 6$ & 10 & MCP \\
\hline 11 & Male & 59 & Tongue SCC & Tongue and FOM & $6 \times 8$ & 9 & MCP \\
\hline 12 & Male & 58 & Tongue SCC & Tongue and FOM & $5 \times 6$ & 9 & MCP \\
\hline 13 & Male & 60 & Tongue SCC & Tongue and FOM & $5 \times 7$ & 8 & MCP \\
\hline 14 & Male & 26 & Tongue SCC & Tongue and FOM & $5 \times 7$ & 8 & MCP \\
\hline 15 & Male & 50 & Tongue SCC & Tongue and FOM & $6 \times 9$ & 9 & IMSP \\
\hline 16 & Male & 33 & Tonge base SCC & Tongue and FOM & $5 \times 7$ & 8 & MCP \\
\hline 17 & Male & 70 & Neck relapse of Ca larynx & Neck skin & $8 \times 12$ & 9 & MCP \\
\hline 18 & Male & 57 & Retromolar trigone SCC & Retromolar trigone & $6 \times 10$ & 11 & MCP \\
\hline 19 & Female & 48 & Tongue SCC & Tongue and FOM & $6 \times 10$ & 8 & MCP \\
\hline 20 & Female & 19 & Facial soft tissue sarcoma & Facial skin & $6 \times 8$ & 13 & MCP \\
\hline
\end{tabular}

SCC: squamous cell carcinoma; Ca: cancer; FOM : floor of mouth; IMSP : intermuscular septal perforator; MCP : musculocutaneous perforator.

Generally, anastomose of one artery and one vein is enough.

Monitoring after flap operation: Close observation for at least $72 \mathrm{~h}$ after flap transplantation is necessary. The patency for vessels after anastomosis was judged by examining the color, elasticity and capillary filling of skin, as well as capillary hemorrhage after acupuncture. If necessary, the blood flow situation was assessed objectively using bedside Doppler ultrasonography.

\section{Results}

Application and anatomy of ALT flap. Application and anatomy of ALT flap in this study is shown in Table 1. The length of the dissected vascular pedicle was ranged from 5 to $14 \mathrm{~cm}$ with an average length of $9.9 \mathrm{~cm}$. The area of ALT cut was $(4-9 \mathrm{~cm}) \times(6-$ $16 \mathrm{~cm})$. All flaps had skin perforators. The types of skin perforators included four cases (20\%) of intermuscular septum perforators and six cases of (80\%) musculocutaneous perforators. ALT flap was used for repairing head and neck defects, including 14 cases of oral mucosa defects (repairing tongue and mouth at the end in nine cases, oral mucosa in three cases, hard palate in one case, and the posterior molars mucosa in one case), and facial and neck skin defects in six cases.

Success ratio and complications. Twenty cases receiving reconstruction of soft tissue defects of the head and neck using free ALT flap were all successful. No complications of skin flap necrosis occurred. One case showed postoperative vascular crisis. After exploratory surgery to ascertain the crisis, anastomosis was performed again and the flap survived. All patients had primary suture at their donor site, except for one case whose abdominal skin had to be cut due to the large size of the donor site. Wound dehiscence in the donor site was observed in one patient, who had a per secundum intentionem. No patients had complications of motor or sensory dysfunction in lower extremity.

\section{Discussion}

The application of ALT flap in the reconstruction of head or neck soft tissue defects achieves a high success rate and has little influence on the donor site. Thus, this technique is a valuable and potential choice, which is worth further research.

The reason that application of ALT flap has been gradually accepted is because of its unique 
characteristics. ALT flap is a perforator flap, whose flap blood supply is from definite small skin perforators. Cutting the perforator flap does not need to damage the vascular trunk, and the flap can survive without attaching to the muscles beneath it. When the flaps are cut off, the motor nerve is preserved which controls the muscles beneath the flap. Additionally, the flap can be primarily sutured after dissection. Therefore, the perforator flap seldom disturbs the shape and functions of the donor site, thus causes less complications. The concept of perforator flap was raised in the 90s and then was developed rapidly, which have become the new developing direction of flap surgery. ${ }^{1,2}$

The history of using ALT flap started from 1984, and Koshima et al. ${ }^{4}$ applied it in the reconstruction of head and neck soft tissue defects in 1993. There are a lot of reports about the application of ALT perforator flap in Western countries, but not in China. Wei et al. $^{3}$ reported the largest number of patients using ALT flap in Taiwan, and they achieved a success rate of $96.4 \%$ among 469 cases. They believe that the ALT perforator flap is an ideal flap donor site for reconstruction of head and neck defects. ALT perforator flap has been used in a variety of head and neck soft tissue defects, including defects in mouth cheek, ${ }^{5}$ craniofacial soft tissue defects, ${ }^{6}$ hypopharynx and cervical esophageal defects and oral soft tissue defects. ${ }^{7}$ There are only a few reports about ALT flap used in head and neck reconstruction in China, most of which were used in the reconstruction of oral and facial soft tissue defects, ${ }^{9-13}$ only a few were in repairing the hypopharynx or the skull base defects.

There are many anatomic variability in the perforators of ALT flap, which is one of the reasons that flap can not be used generally. The results from this article revealed that intermuscular septum perforators account for $20 \%$, while musculocutaneous perforators account for $80 \%$. Although the proportion of different types of perforator is different as reported, it is more complicated to harvest the flap with musculocutaneous perforator. ${ }^{14,15}$ Surface markers and two-dimensional color ultrasonic Doppler were coordinately applied preoperatively to locate the perforator in this group of patients, which determined whether the perforators existed and where the perforators passed through. We believe that this would help the design and harvest of the flap. Tsukino et al. ${ }^{16}$ has compared the accuracy between the pen-based Doppler detector and color ultrasonic Doppler used to detect perforators of ALT. The coincidence was only $40 \%$ between results from the pen-based Doppler detector and results found in operation, but was $100 \%$ with color ultrasonic Doppler. They believe that the color Doppler ultrasound is more accurate than acoustic Doppler for detecting the location where the perforator passes out. In this study, small blood vessels which are $3 \mathrm{~mm}$ beneath the skin could be explored using the highfrequency color ultrasonic Doppler, and the lateral circumflex femoral artery could be detected when scanning in deeper layers. The passing-out points of the perforator could be determined by this method.

In this article, the size of incised ALT flaps were between 4-9 cm and 6-16 cm, which can be cut and shaped easily, and generally sufficient for reconstruction of head and neck defects. The lengths of the vascular pedicle were ranged from $5 \mathrm{~cm}$ to $14 \mathrm{~cm}$ with an average of $14 \mathrm{~cm}$, which can lead the vascular pedicle to the recipient site and make vascular anastomosis convenient. No cases were found to have insufficient length of vascular pedicle. Furthermore, the lacouna of head and neck defects can be repaired by slightly thicker ALT. The advantages discussed above make it quite proper to use ALTs to repair and reconstruct head and neck defects.

Reconstruction using ALT flaps succeeded in all 20 cases mentioned in this article, which is similar to the success ratio of above $95 \%$ in other reports. Although one case showed post-operative vascular crisis, it was retrieved through re-exploration. This demonstrates that using such flaps can obtain pretty great operational success ratios. Besides, it was reported that ALT flaps whose width is no more than $9 \mathrm{~cm}$ can receive primary suture. In the current study, only two cases needed second intention in the wound surface of the donor site. Therefore, using ALT flaps seldom influences the donor site.

At present, the number of cases using free ALT flaps for repairing head and neck defects is small, and there are only 20 cases reported in this article. Reconstruction of soft tissue defects in head and neck mainly involved the mucous membrane of mouth and the skins of face and neck, but not the complicated defects, such as the basilar region or esophagus in the cervical segments. There are also some limits in our research. The recovery of the post-operative functions and the commonly used 
comparative analysis of forearm flap are not studied in this article. In addition, because the ALT flap is slightly thicker than the forearm flap, it is usually used for the small oral tissues defects, especially when bucca cavioris defects become swollen. How to slice the flap thinner to meet the need of small tissue defects needs further investigation. In sumary, we believe that, although there are still many questions to be solved, the ALT flap is a considerably ideal donor site for the reconstruction of head and neck defects.

\section{References}

[1] Zhang SM, Xu DC, Gu YD. Perforator flap[J]. Zhong Guo Lin Chuang Jie Pou Xue Za Zhi, 2004, 22(1): 32-35. [in Chinese]

[2] Zhang B. Application of perforator flaps in Head and Neck[J]. Zhonghua Er Bi Yan Hou Tou Jing Wai Ke Za Zhi, 2006, 41 (11): 878-880. [in Chinese]

[3] Wei FC, Jain V, Celik N, et al. Have we found an ideal softtissue flap? An experience with 672 anterolateral thigh flaps [J]. Plast Reconstr Surg, 2002, 109(7):2219-2226.

[4] Koshima I, Hosoda M, Moriguchi T, et al. A combined anterolateral thigh flap, anteromedial thigh flap, and vascularized iliac bone graft for a full-thickness defect of the mental region [J]. Ann Plast Surg, 1993,31(2) : 175-180.

[5] Ozkan O, Mardini S, Chen HC, et al. Repair of buccal defects with anterolateral thigh flaps $[\mathrm{J}]$. Microsurgery, 2006, 26 (3) : 182-189.

[6] Amin A, Rifaat M, Civantos F, et al. Free anterolateral thigh flap for reconstruction of major craniofacial defects $[\mathrm{J}]$. J Reconstr Microsurg, 2006,22(2) :97-104.

[7] Yu P, Robb GL. Pharyngoesophageal reconstruction with the anterolateral thigh flap: a clinical and functional outcomes study $[\mathrm{J}]$. Plast Reconstr Surg, 2005, 116(7) : 1845-1855.

[8] Shieh SJ, Chiu HY, Yu JC, et al. Free anterolateral thigh flap for reconstruction of head and neck defects following cancer ablation [J]. Plast Reconstr Surg, 2000, 105(7) :2349-2357.

[9] Xu GP, Liu JC, Zeng ZY, et al. Microvascular anastomotic anterolateral thigh flap for reconstruction of complicated head and neck defects following cancer ablation [J]. Zhonghua Xian Wei Wai Ke Za Zhi, 2002, 25 (3): 177-179. [in Chinese]

[10] Huang WX, Chen J, Yu JJ, et al. Tongue Reconstruction with Free Anterolateral Thigh Flap in 21 Cases with Tongue Carcinoma[J]. Zhong Guo Zhong Liu, 2006, 15(11):779-781. [in Chinese]

[11] Chen J, Huang WX, Zhou X, et al. Free anterolateral thigh flap for repairing the defects of oral cavity and maxillofacial $[\mathrm{J}]$. Zhonghua Er Bi Yan Hou Tou Jing Wai Ke Za Zhi, 2006,41(8): 587-590. [in Chinese]

[12] Zhang B, Li DZ, Xu ZG, et al. Free anterolateral thigh flap for reconstruction of head and neck defects [J]. Zhonghua Er Bi Yan Hou Tou Jing Wai Ke Za Zhi, 2006,41(6):447-450. [in Chinese]

[13] Li H, Wu GH, Liu JC, et al. The application of anterolateral thigh flap to reconstruct head and neck defects [J]. Zhonghua Xian Wei Wai Ke Za Zhi, 2006,29(2):146-147. [in Chinese]

[14] Valdatta L, Tuinder S, Buoro M, et al. Lateral circumflex femoral arterial system and perforators of the anterolateral thigh flap: an anatomic study [J]. Ann Plast Surg, 2002,49(2): $145-150$.

[15] Kimata Y, Uchiyama K, Ebihara S, et al. Anatomic variations and technical problems of the anterolateral thigh flap: a report of 74 cases [J]. Plast Reconstr Surg, 1998,102 (5) : 1517 1523.

[16] Tsukino A, Kurachi K, Inamiya T, et al. Preoperative color Doppler assessment in planning of anterolateral thigh flaps $[\mathrm{J}]$. Plast Reconstr Surg, 2004,113(1) :241-246. 